\title{
Mitofusin-2 Expression Is Implicated in Cervical Cancer Pathogenesis
}

\author{
SUNG YONG AHN ${ }^{1}$, CHENGRI LI $^{2}$, XIANGLAN ZHANG ${ }^{3}$ and YOUNG-MIN HYUN ${ }^{1,4}$ \\ ${ }^{1}$ Department of Anatomy and ${ }^{4}$ BK21 PLUS Project for Medical Science, \\ Yonsei University College of Medicine, Seoul, Republic of Korea; \\ ${ }^{2}$ Oral Cancer Research Institute, Yonsei University College of Dentistry, Seoul, Republic of Korea; \\ ${ }^{3}$ Department of Pathology, Yanbian University Hospital, Yanji, P.R. China
}

\begin{abstract}
Background/Aim: It remains unclear whether mitofusin-2 (MFN2) functions as a tumour suppressor or oncogene in cancer progression. In this study we, therefore, aimed to investigate the effect of MFN2 on the pathogenesis of cervical cancer. Materials and Methods: MFN2 expression was detected in seven healthy cervical, 64 cervical intraepithelial neoplasia (CIN), and 120 cervical squamous cell carcinoma (SCC) tissues by immunohistochemistry. Moreover, biological function of MFN2 in cervical cancer was investigated in vitro. Results: MFN2 levels exhibited a tendency to gradually increase from healthy cervical tissue to CIN to SCC. Moreover, MFN2 expression was significantly associated with higher T-stage $(p=0.008)$ and lymph node metastasis $(p<0.001)$. The proliferative, migratory, and invasive abilities of MFN2-knockdown cells were significantly lower $(p<0.001, p<0.001$, and $p<0.001$, respectively) than control cells. Conclusion: MFN2 may be involved in cervical cancer pathogenesis as an oncogene and might serve as a biomarker of cervical SCC.
\end{abstract}

Cervical cancer is one of the most common gynaecological malignant tumours (1-4) and the second most common cancer, after breast cancer, among women worldwide (3). According to the World Health Organization, approximately

Correspondence to: Sung Yong Ahn, Ph.D., Department of Anatomy, Yonsei University College of Medicine, 50-1 Yonsei-Ro, Seodaemun-gu, Seoul 03722, Republic of Korea. Tel: +82 222281666, Fax: +82 23650700, e-mail: sungyong@yuhs.ac and Young-Min Hyun, Ph.D., Department of Anatomy and BK21 PLUS Project for Medical Science, Yonsei University College of Medicine, 50-1 Yonsei-Ro, Seodaemun-gu, Seoul 03722, Republic of Korea. Tel: +82 222281655, Fax: +82 23650700, e-mail: ymhyun@yuhs.ac

Key Words: Mitofusin-2, oncogene, cervical intraepithelial neoplasia, cervical carcinogenesis, cervical cancer pathogenesis, biomarker.
528,000 cervical cancer cases were diagnosed and 266,000 deaths were attributed to this malignancy globally in 2012 $(1,2)$. The prognosis associated with cervical cancer remains poor, and the molecular mechanism underlying its progression is largely unknown.

Over recent years, several biomarkers useful in the diagnosis and treatment of cervical cancer have emerged. For instance, human papillomavirus DNA is a well-known diagnostic and therapeutic molecular biomarker of cervical cancer $(5,6)$, and certain protein-based biomarkers, such as squamous cell carcinoma (SCC) antigen (7, 8), carcinoembryonic antigen (9-11), and members of the matrix metalloproteinase family, are also used in diagnosing cervical cancer $(12,13)$. However, further research is needed to identify biomarkers of greater sensitivity in order to improve diagnostic accuracy and therapeutic efficacy.

Mitofusin-2 (MFN2) is a mitochondrial outer membrane protein that plays an essential role in mitochondrial fusion and contributes to maintenance and action of the mitochondrial network (14-16). MFN2 is a well-known hyperplasia-suppressor gene, and was initially identified in the vascular smooth muscle cells of hypertensive rats (17). In addition to hypertension, dynamic interactions between abnormal MFN2 expression and the pathogenesis of various disorders, such as obesity (18), Charcot-Marie-Tooth disease (19), atherosclerosis (20), and diabetes (21), have been demonstrated in previous investigations.

Recent research indicated crucial roles for MFN2 in cancer progression; however, the question of whether it acts as a tumour suppressor or oncogene in this process remains controversial. Several studies supported the hypothesis that MFN2 is a tumour suppressor; for example, its expression has been shown to be lower in hepatocellular carcinoma, bladder cancer, and gastric cancer tissues than in corresponding healthy tissue samples (22-24). Moreover, MFN2 overexpression in cells of urinary bladder carcinoma lines can induce cell-cycle arrest and apoptosis, thereby inhibiting cell proliferation (23). Similarly, its overexpression 
can restrict the proliferative, colony-forming, migratory, and invasive abilities of cells of gastric cancer lines (24). In contrast, other studies have provided evidence that MFN2 functions as an oncogene in gastric cancer (25). Moreover, in vitro experiments have demonstrated that MFN2 knockdown exerts inhibitory effects on lung cancer cell proliferation, migration, and invasion (26). Thus, the impact of MFN2 on carcinogenesis and cancer progression is likely to be more complicated than expected and further research is needed.

The role of MFN2 in cervical cancer has not yet been examined as far as we are aware. In the present work, we therefore investigated the relevance of MFN2 expression to the clinicopathological characteristics of patients with cervical cancer and also assessed the influence of MFN2 knockdown on the behaviour of cells of cervical cancer lines, with the aim of determining the implications of MFN2 expression in cervical cancer pathogenesis.

\section{Materials and Methods}

Clinical materials. Commercially available tissue microarrays (TMAs), including 120 cervical SCC, 64 cervical intraepithelial neoplasia (CIN), and seven healthy cervical tissue samples (catalogue numbers CIN482, CIN483, CXC1501, and CXC1502), were purchased from Pantomics, Inc. (Richmond, CA, USA). The characteristics of the patients whose tissues were included in the present work are presented in Table I. This study was approved by the Institutional Review Board of Yonsei University Health System, Severance Hospital (IRB No. 4-2017-1164). Written consent was obtained from all enrolled patients for use of tissue specimens.

Immunohistochemistry. The TMA sections were deparaffinized with xylene and hydrated using a graded ethanol series. Endogenous peroxidase activity was inhibited using a $1: 40 \mathrm{H}_{2} \mathrm{O}_{2}$ : methanol mixture before antigen retrieval was performed with an antigen retrieval solution (Dako, Carpinteria, CA, USA) and the pressure cooker method. The sections were then exposed to a mouse monoclonal IgG primary antibody to MFN2 (Abcam, Cambridge, MA, USA) diluted 1:200, and a REAL EnVision HRP Rabbit/Mouse Detection System (Dako) secondary antibody. Visualization of antibody binding was performed using the chromogen 3,3'-diaminobenzidine, and the tissues were then counterstained with haematoxylin. Mouse IgG (DakoCytomation Denmark A/S, Glostrup, Denmark) was used as a negative control. A cell block was constructed using HeLa cells (ATCC, Rockville, $\mathrm{MD}$, USA), sections of which were then made for use as positive controls (Figure 1A).

As described in our previous study, the weighted histoscore method was used to score MFN2 expression according to staining intensity and the percentage of positively stained cells (27). The histoscore was calculated as follows: final score $=(0 \times$ percentage of negative cells $)+(1 \times$ percentage of light-brown cells $)+(2 \times$ percentage of brown cells $)+(3 \times$ percentage of dark-brown cells). The samples were subsequently divided into two groups according to the final histoscore into low expression (histoscores from 0 through 100) and high expression (histoscores from 101 through 300).
Table I. Summary of characteristics for all clinical samples used in this study.

\begin{tabular}{lc}
\hline Clinicopathological variable & Value \\
\hline Healthy cervical tissues & \\
Total no. of cases & 7 \\
Median age (range), years & $41(38-51)$ \\
CIN & 64 \\
Total no. of cases & $46(23-72)$ \\
Median age (range), years & \\
Grade, n (\%) & $22(34.4)$ \\
I & $26(40.6)$ \\
II & $16(25.0)$ \\
III & 120 \\
SCC & $45(22-70)$ \\
Total no. of cases & $109(90.8)$ \\
Median age (range) & $11(9.2)$ \\
T-Stage, n (\%) & $89(74.2)$ \\
T1 & $31(25.8)$ \\
T2-4 & \\
LN metastasis, n (\%) & \\
Absent & Present \\
\hline
\end{tabular}

CIN: Cervical intraepithelial neoplasia; LN, lymph node; SCC: squamous cell carcinoma.

Cell lines and culture. Human cervical cancer cells of the HeLa (ATCC) and SiHa (ATCC) lines were used in this study. These cells were cultured in Roswell Park Memorial Institute 1640 medium (Nissui Pharmaceutical Co., Ltd., Tokyo, Japan) supplemented with $10 \%$ foetal bovine serum (Invitrogen, Carlsbad, CA, USA), $100 \mathrm{U} / \mathrm{ml}$ penicillin, and $0.1 \mathrm{mg} / \mathrm{ml}$ streptomycin-amphotericin B (Lonza, Basel, Switzerland). MFN2 expression was down-regulated in these cells by transfection of small-interfering RNA (siRNA) (Bioneer, Seoul, South Korea) using Lipofectamine 2000 (Invitrogen) according to the manufacturer's instructions. Knockdown efficiency was evaluated by reverse-transcription real-time quantitative polymerase chain reaction (RT-qPCR) and western blotting.

$R T$-qPCR. Total RNA was isolated from HeLa and $\mathrm{SiHa}$ cells following transfection with scrambled (Scr)-siRNA or MFN2-siRNA using TRIzol Reagent (Invitrogen), and complementary DNA synthesis was performed using oligo(dT) primer, recombinant RNasin ribonuclease inhibitor, and Moloney murine leukaemia virus reverse transcriptase (Promega, Madison, WI, USA). Marker of proliferation Ki-67 (MKI67), proliferating cell nuclear antigen (PCNA), and MFN2 mRNA expression was determined by qPCR using $2 \times$ SYBR Premix Ex Taq II (Tli RnaseH Plus) (RR82LR; Takara, Ann Arbor, MI, USA) on an Applied Biosystems (Foster City, CA, USA) instrument. The following primers were used: MKI67 forward: 5'-AAGCCC TCCAGCTCCTAGTC-3' and reverse: 5'-GCAGGTTGCCACTCT TTCTC-3'; PCNA forward: 5'-GGCGTGAACCTCACC AGTAT-3' and reverse: 5'-TTCTCCTGGTTTGGTGCTTC-3'; $\beta$-actin forward: 5'-ATAGCACAGCCTGGATAGCAACGTAC-3' and reverse: 5'CACCTTCTACAATGAGCTGCGTGTG-3'. The relative expression of each gene was normalized to that of actin, and data analysis was performed using the $\Delta \Delta \mathrm{Cq}$ method. 

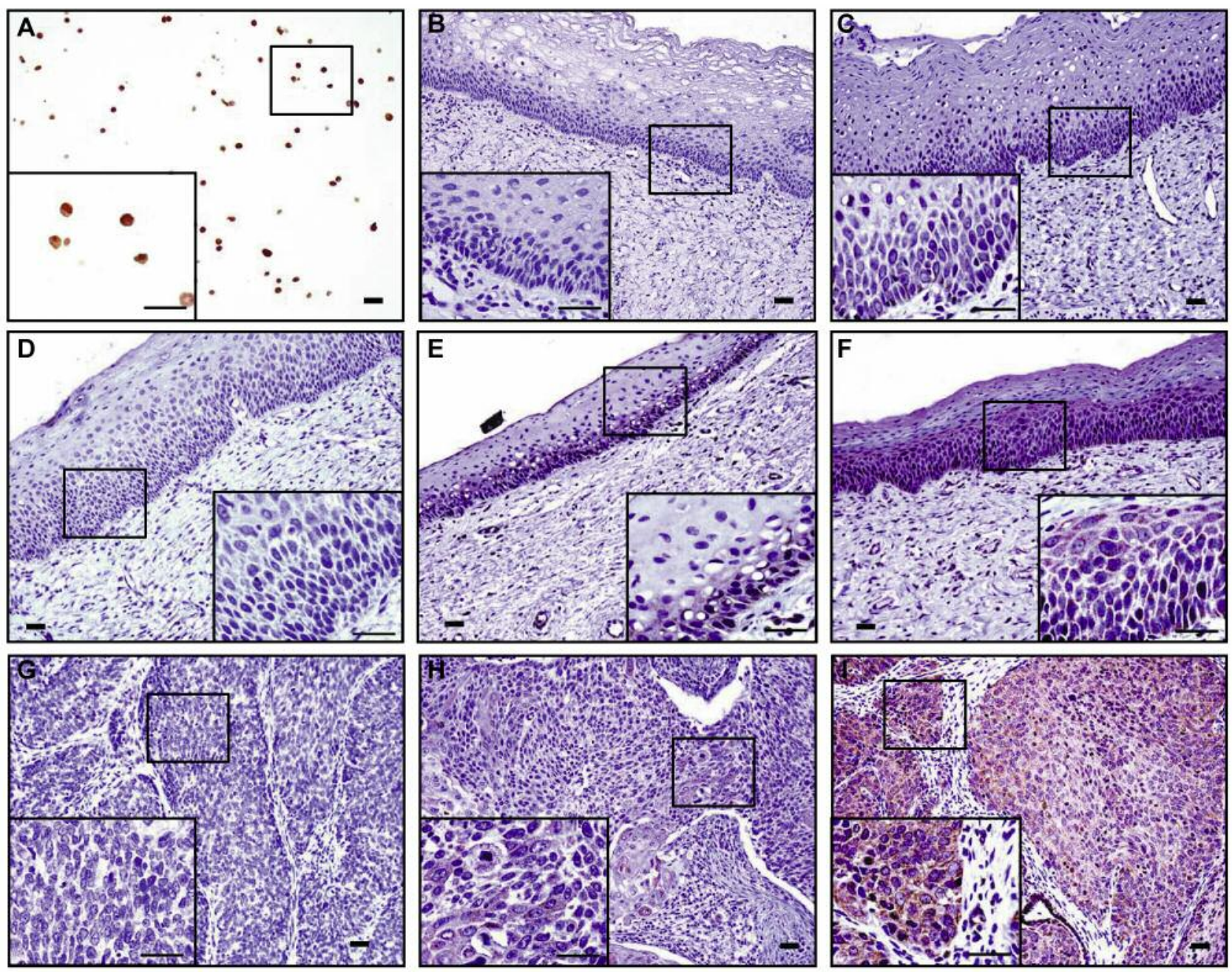

Figure 1. Representative expression patterns for mitofusin-2 (MFN2) in cervical tissue sections. A HeLa cell block was used as positive control (A). Normal cervical tissues showed negative (B) or a low level (C) of MFN2 expression. By contrast, negative, low, and high levels of MFN2 expression were found in cervical intraepithelial neoplasia $(D-F)$, and cervical squamous cell carcinoma tissue samples $(G-I)$. Original magnification, main image: $\times 200$; inset $\times 1,000$; scale bar $=20 \mu \mathrm{m}$.

Western blotting. Protein samples were extracted from Scr-siRNA or MFN2-siRNA transfected HeLa and SiHa cells using cell lysis buffer (Cell Signaling Technology, Danvers, MA, USA). The protein samples were resolved by sodium dodecyl sulfate-polyacrylamide gel electrophoresis and subjected to immunoblotting. The proteins were transferred to nitrocellulose membranes (1620115; Bio-Rad, Hercules, CA, USA), which were then blocked and incubated at room temperature for $1 \mathrm{~h}$ with primary antibodies against MFN2 (diluted 1 in 1000; Abcam, Cambridge, UK) and $\beta$-actin (diluted 1 in 1000; Cell Signaling Technology). After incubation with the corresponding secondary antibodies, protein bands were visualized using an enhanced chemiluminescence detection system (Pierce Biotechnology Inc., Rockford, IL, USA).

Cell proliferation assay. To investigate the effect of MFN2 expression on cell proliferation, the number of viable HeLa and $\mathrm{SiHa}$ cells was counted at different time points following transfection with Scr-siRNA or MFN2-siRNA. Cells were seeded in a 6-well plate at a density of $1 \times 10^{5}$ and counted after trypan blue staining each day for 3 days.

Wound-healing and invasion assays. The impact of MFN2 expression on cell migration and invasion was assessed by subjecting cells transfected with Scr-siRNA or MFN2-siRNA to wound-healing and matrigel invasion assays. For the wound-healing assay, the cells were seeded in a 24 -well plate at a density of $5 \times 10^{4}$. After reaching about $90 \%$ confluence, the cells were scratched by a sterile $0.2 \mathrm{ml}$ pipette tip to create wounds, and relative wound closure was determined after 18 and $24 \mathrm{~h}$ of scratch wounding. The matrigel invasion assay was performed using transwell chambers with a pore size of $8 \mu \mathrm{m}$ (BD Biosciences, Bedford, MA, USA) coated with $8 \mu \mathrm{g} / \mu \mathrm{l}$ matrigel (BD Biosciences, San Jose, CA, USA). The cells were seeded in the upper chamber of transwell at a density of $5 \times 10^{4}$ with culture medium containing $3 \%$ BSA and culture 
medium containing $10 \%$ FBS was added in the bottom chamber. After $34 \mathrm{~h}$ of culture, the lower side of each membrane was stained with $0.25 \%$ crystal violet, and the number of invading cells was counted under a microscope.

Statistical analysis. The chi-squared test and Fisher's exact test were used to examine the association between MFN2 protein expression and clinicopathological parameters. Differences between groups of cells in the proliferation, migration, and invasion assays were analysed using the Mann-Whitney $U$-test. Differences were considered to be statistically significant when $p<0.05$.

\section{Results}

Clinicopathological characteristics of tissue samples. In total, seven healthy cervical, $64 \mathrm{CIN}$, and 120 cervical SCC tissues were tested. The clinicopathological features of the tissue samples are summarized in Table I.

MFN2 expression in healthy, CIN, and SCC cervical tissue samples. Cytoplasmic expression of MFN2 was observed in three out of seven (42.9\%), 43 out of $64(67.2 \%)$, and 91 out of $120(75.8 \%)$ of the healthy cervical, CIN, and SCC tissue samples, respectively. Representative expression patterns for MFN2 in the different tissues are shown in Figure 1. The healthy cervical tissues were negative or only faintly positive for MFN2 staining, and the positive cells observed were mainly restricted to the basal cell layer (Figure $1 \mathrm{~B}$ and $\mathrm{C}$ ). In contrast, $26.6 \%$ of the CIN tissues exhibited high MFN2 immunoreactivity, with positive cells being predominantly located in epithelium displaying dysplastic changes (Figure1E and F). High MFN2 expression was detected more often among CIN tissues of grades II (34.6\%) and III $(50.0 \%)$ than those of grade I $(0 \%)(p<0.001$ and $p=0.014$, respectively) (Table II). Cancer cells within the SCC tissues examined typically demonstrated cytoplasmic MFN2 expression (Figure $1 \mathrm{H}$ and I), and high expression of MFN2 was observed more often among SCC tissues (50.8\%) than healthy cervical $(0 \%)(p=0.014)$ and CIN tissues $(26.6 \%)$ $(p=0.002)$ (Table II).

We next tested for correlation between MFN2 expression and the clinicopathological characteristics of the 120 patients with cervical SCC. High MFN2 expression was more common among patients with high $\mathrm{T}$ stage $(90.9 \%)$ than those with low T stage $(p=0.008)$, and was also observed more often among patients with lymph node (LN) metastasis $(83.9 \%)$ than those without $(39.3 \%) \quad(p<0.001)$. No significant association was evident between age and MFN2 expression (Table III).

Efficient MFN2 knockdown by siRNA transfection of human cervical cancer cell lines. MFN2 knockdown in cervical cancer cell lines was achieved by siRNA transfection (Figure 2A). Relative to those treated with the Scr-siRNA control,
MFN2 mRNA expression in SiHa cells transfected with $M F N 2$-siRNA was significantly reduced $24 \mathrm{~h}(0.14 \pm 0.08)$ $(p<0.001), 36 \mathrm{~h}(0.21 \pm 0.07)(p<0.001)$, and $72 \mathrm{~h}(0.34 \pm 0.06)$ $(p<0.001)$ after transfection. MFN2-siRNA-transfected HeLa cells also demonstrated significantly decreased MFN2 mRNA expression $24 \mathrm{~h}(0.10 \pm 0.06) \quad(p<0.001), 36 \mathrm{~h}$ $(0.16 \pm 0.06)(p<0.001)$, and $72 \mathrm{~h}(0.31 \pm 0.05)(p<0.001)$ post transfection relative to the corresponding Scr-siRNA-treated control. Moreover, western blotting revealed that in comparison to the Scr-siRNA control, MFN2-siRNA transfection resulted in obviously reduced MFN2 protein expression in both $\mathrm{SiHa}$ and HeLa cells.

MFN2 knockdown attenuates proliferation of cervical cancer cells. Relative to SiHa cells treated with Scr-siRNA, those transfected with MFN2-siRNA were significantly reduced in number 24-72 h after transfection ( $p<0.001$; Figure 2B i). Similar results were obtained using HeLa cells, with MFN2siRNA transfection leading to a decrease in relative cell number 24-72 h post transfection ( $p<0.001$; Figure $2 \mathrm{~B}$ ii). Moreover, both $\mathrm{SiHa}$ and HeLa cells transfected with MFN2siRNA showed significantly decreased expression of MKI67 (both $p<0.001$ ) and PCNA (both $p<0.001$ ) mRNA expression relative to the Scr-siRNA groups $24 \mathrm{~h}$ after transfection (Figure 2B iii and iv).

MFN2 knockdown attenuates cervical cancer cell migration and invasion. A wound-healing assay was performed to investigate the effect of MFN2 on cervical cancer cell migration. Relative to the corresponding control groups,

Figure 2. Knockdown of mitofusin-2 (MFN2) expression by MFN2siRNA transfection in cervical cancer cell lines at different time points. A: The protein ( $i$ and iii) and $m R N A$ (ii and iv) expression of MFN2 were compared between MFN2-siRNA-treated cells and scrambled control SiHa (i and ii) and HeLa (iii and iv) cell lines over time by western blot and real-time polymerase chain reaction analysis. B: Proliferative ability was significantly reduced in MFN2 knockdown cells compared to the scrambled control cervical cancer cell lines SiHa (i) and HeLa (ii). Marker of proliferation Ki-67 (MKI-67) (iii) and proliferating cell nuclear antigen (PCNA) (iv) mRNA expression was significantly reduced in MFN2 knockdown cells compared to the scrambled control in SiHa and HeLa cell lines. C: Cell motility was significantly reduced in MFN2-knockdown cells compared to scrambled control both in SiHa (i) and HeLa (ii) cell lines (original magnification, $\times 100$; scale bar $=100 \mu \mathrm{m})$. D: Invasive ability of the cells was significantly reduced in MFN2 knockdown cells compared to the scrambled control in cervical cancer cell lines in both SiHa (i) and HeLa (ii) cell lines (original magnification, $\times 100$; scale bar $=100 \mu \mathrm{m}$ ). Quantitative results indicate average values $\pm S D$ of three independent experiments, each of which was conducted in triplicate $(n=9)$. The results were analyzed by the Mann-Whitney U-test. *Significantly different at $p<0.001$. 
$\mathbf{A}_{\text {(i) SiHa }}$

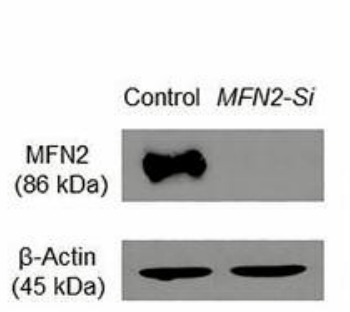

B

(i) $\mathrm{SiHa}$

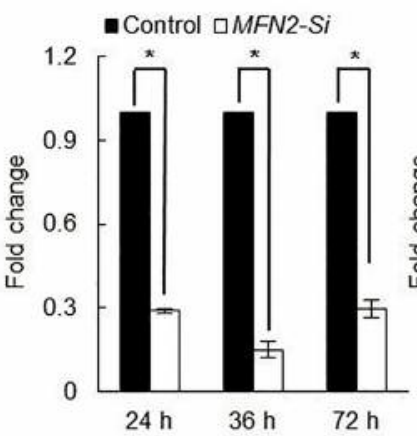

C (i)

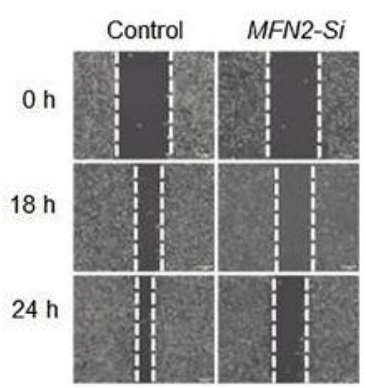

$\mathbf{D}_{\text {(i) }}$

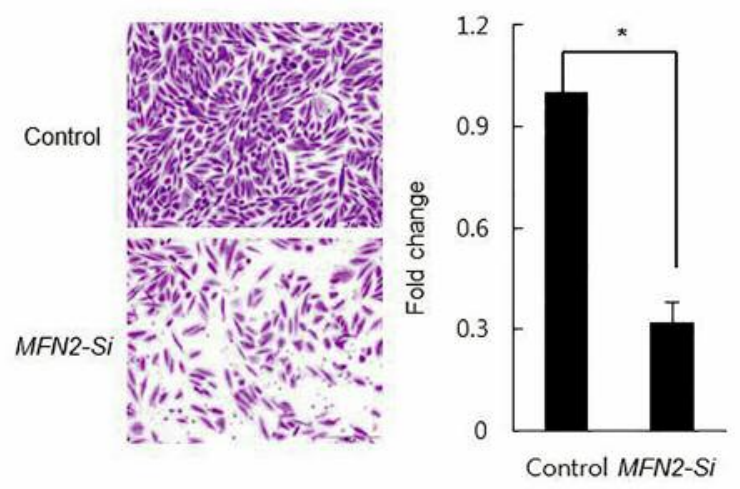

(ii) HeLa (iii) HeLa

(iv)
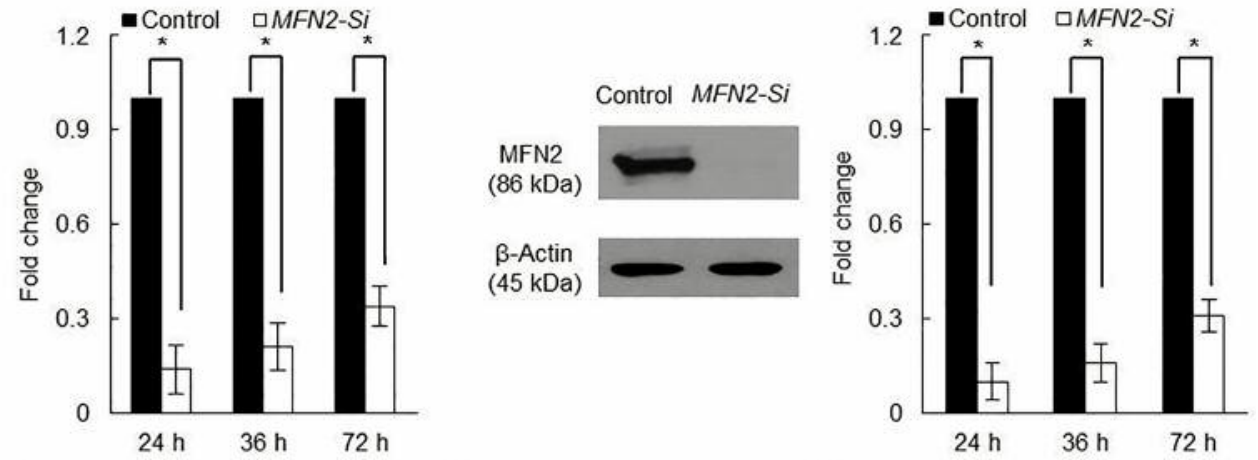

(iii)

(iv)
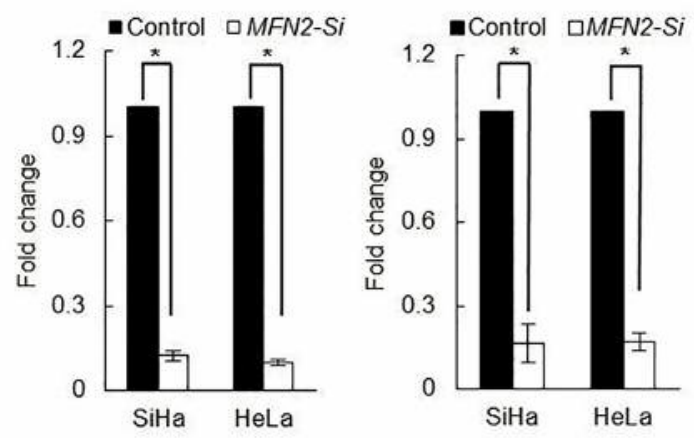

(ii)
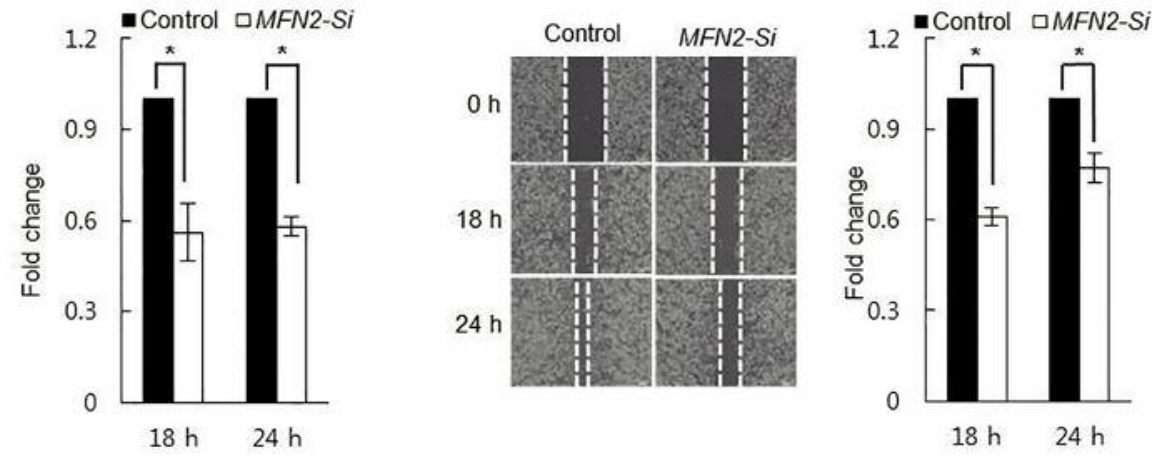

(ii)
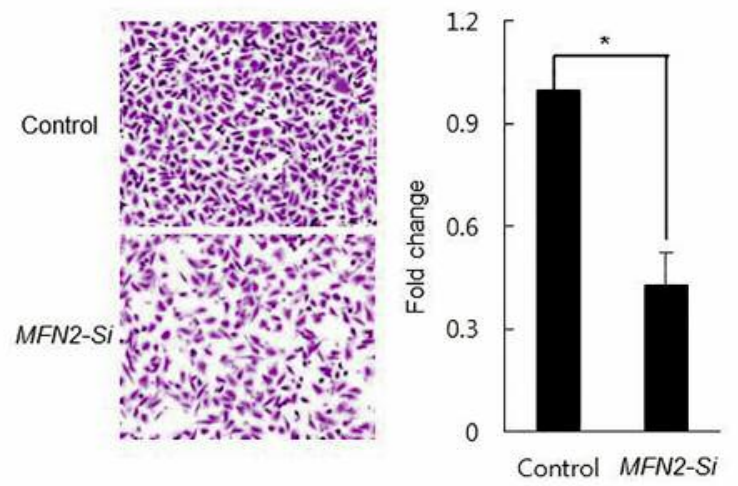
Table II. Mitofusin-2 (MFN2) expression in healthy cervix, cervical intraepithelial neoplasia (CIN), and cervical squamous cell carcinoma (SCC) tissues.

\begin{tabular}{|c|c|c|c|c|}
\hline \multirow[t]{2}{*}{ Tissue type } & \multirow[t]{2}{*}{ No. of cases } & \multicolumn{2}{|c|}{ MFN2, n (\%) } & \multirow[t]{2}{*}{$p$-Value } \\
\hline & & Low & High & \\
\hline Healthy cervical tissue & 7 & $7(100)$ & $0(0)$ & \\
\hline CIN & 64 & $47(73.4)$ & 17 (26.6) & \\
\hline I & 22 & $22(100)$ & $0(0)$ & \\
\hline II & 26 & $17(65.4)$ & $9(34.6)$ & $0.002 v s$. CIN I \\
\hline III & 16 & $8(50.0)$ & $8(50.0)$ & $<0.001 v s$. CIN I \\
\hline \multirow[t]{3}{*}{$\mathrm{SCC}$} & 120 & $59(49.2)$ & $61(50.8)$ & $0.014 v s$. normal \\
\hline & & & & $0.002 v s . \mathrm{CIN}$ \\
\hline & & & & $<0.001$ vs. CIN I \\
\hline
\end{tabular}

Table III. Clinicopathological significance of mitofusin-2 (MFN2) expression in patients with cervical cancer.

\begin{tabular}{|c|c|c|c|c|}
\hline \multirow[t]{2}{*}{ Variable } & \multirow[t]{2}{*}{ No. of cases, $(\%)$} & \multicolumn{2}{|c|}{ MFN2 } & \multirow[t]{2}{*}{$p$-Value } \\
\hline & & Low & High & \\
\hline \multicolumn{5}{|l|}{ Age } \\
\hline$<45$ Years & $59(49.2)$ & $30(51.7)$ & $28(48.3)$ & 0.588 \\
\hline$\geq 45$ Years & $61(50.8)$ & $29(46.8)$ & $33(53.2)$ & \\
\hline \multicolumn{5}{|l|}{ T-Stage } \\
\hline $\mathrm{T} 1$ & $109(90.8)$ & $58(53.2)$ & $51(46.8)$ & 0.008 \\
\hline $\mathrm{T} 2-4$ & $11(9.2)$ & $1(9.1)$ & $10(90.9)$ & \\
\hline \multicolumn{5}{|l|}{ LN metastasis } \\
\hline Absent & $89(74.2)$ & $54(60.7)$ & $35(39.3)$ & $<0.001$ \\
\hline Present & $31(25.8)$ & $5(16.1)$ & $26(83.9)$ & \\
\hline
\end{tabular}

LN, Lymph node.

transfection of MFN2-siRNA significantly suppressed the migration of both SiHa and HeLa cells, as measured $18 \mathrm{~h}$ (both $p<0.001$ ) and $24 \mathrm{~h}$ (both $p<0.001$ ) after scratch wounding (Figure $2 \mathrm{C}$ I and ii). In addition, a matrigel invasion assay was performed to explore the influence of MFN2 on cervical cancer cell invasion. The number of MFN2-siRNAtransfected SiHa and HeLa cells having traversed the membrane was found to be significantly lower (both $p<0.001$ ) relative to the control groups (Figure 2D I and ii).

\section{Discussion}

SCC is the most common histological type of cervical cancer and develops via a multistep process, whereby healthy squamous epithelium undergoes dysplastic changes, termed CIN, that are followed by cancer formation. In the present study, we compared MFN2 levels in healthy, CIN, and SCC cervical tissue samples, with the aim of evaluating changes in MFN2 expression during cervical carcinogenesis. We found that MFN2 expression tended to increase gradually from healthy tissues to $\mathrm{CIN}$ and to SCC, indicating that it may be involved in cervical cancer progression. Moreover, we also found an association between CIN grade and MFN2 expression, which increased from grade I to II and III, implying that MFN2 may also be crucial in the malignant transformation of CIN. Our results are consistent with previous studies that showed that MFN2 expression is significantly higher in lung cancer tissues than in adjacent healthy tissues (26). Furthermore, significantly increased MFN2 expression has been documented in gastric cancer tissues compared to non-tumour tissues (25), and also positively correlated with depth of invasion, clinical stage, and vascular invasion in this cancer type (25). Similar oncogenic effects were also noted in the current work, in which a significant relationship between increased MFN2 expression and poor prognostic indicators, such as higher Tstage and LN metastasis, was noted among patients with cervical SCC.

However, MFN2 has also been reported to act as a tumour suppressor in various cancer types (22-24). In addition to 
reports of its down-regulation in cancer tissues compared to corresponding healthy tissues, a reduced level of MFN2 has been identified as a poor prognostic indicator in several types of cancer, including hepatocellular carcinoma $(22,28)$, breast cancer (29), and lung cancer (29). Moreover, MFN2 overexpression in cells of various malignancies significantly reduced their proliferative, migratory, and invasive abilities (22-24). Such observations are clearly inconsistent with the notion that MFN2 functions as an oncogene. In the present study, the proliferation, migration, and invasion of cervical cancer cells were reduced in cells in which MFN2 expression had been knocked-down. In a previous investigation in A549 lung cancer cells, knockdown of MFN2 was found to exert similar significant effects on proliferation, migration, and invasion (26). The role of MFN2 in cancer development may largely depend on the cancer type or microenvironmental conditions in question. Although a definitive characterization of $M F N 2$ as an oncogene or tumour suppressor remains elusive, the above findings consistently point towards a crucial role for this protein in cancer progression.

Data concerning the molecular basis for the function of MFN2 in cancer are limited. Microarray analysis demonstrated that MFN2 knockdown in A549 cells altered expression of several tumour-associated genes, such as ras-related protein 1A $(R A P 1 A)$, ras-like proto-oncogene $\mathrm{B}(R A L B)$, and integrin subunit alpha 2 (ITGA2) (26). These three genes exert tumourpromoting effects in various malignancies by affecting cell adhesion, proliferation, migration, and survival, as well as metastasis (30-32). Moreover, cell-cycle and DNA replication pathways and extracellular matrix-receptor interaction were identified as being significantly over-represented in a functional pathway enrichment analysis of MFN2-knockdown A549 cells (26). Further study may be needed to precisely establish the molecular mechanisms underlying the effects of MFN2 in cancer.

In summary, we found that MFN2 expression is significantly associated with indicators of poor prognosis among patients with cervical cancer and has a strong influence on the behaviour of cervical cancer cells in vitro. MFN2 expression may thus be involved in cervical cancer pathogenesis. These findings also provide evidence that MFN2 may serve as a molecular biomarker in this malignancy. Nevertheless, further investigation of the role of MFN2 in cervical cancer progression using a larger cohort of patients is needed, and the molecular mechanisms by which MFN2 affects cervical oncogenesis also require clarification.

\section{Funding}

This study was supported by a grant from the National Research Foundation of Korea (NRF-2017R1A6A3A11029376; S.Y.A) and a faculty research grant of Yonsei University College of Medicine (62016-0132;Y-M.H.).

\section{Ethics Approval and Consent to Participate}

For the use of human samples, approval was obtained by the Institutional Review Board of Yonsei University Health System, Severance Hospital (IRB No. 4-2017-1164). Written consent was obtained from all enrolled patients for use of tissue specimens.

\section{Conflicts of Interest}

The Authors declare that they have no competing interests in regard to this study.

\section{References}

1 Jung HS, Rajasekaran N, Ju W and Shin YK: Human papillomavirus: Current and future RNAi therapeutic strategies for cervical cancer. J Clin Med 4(5): 1126-1155, 2015.

2 Torre LA, Bray F, Siegel RL, Ferlay J, Lortet-Tieulent J and Jemal A: Global cancer statistics, 2012. CA Cancer J Clin 65(2): 87-108, 2015.

3 Dasari S, Wudayagiri R and Valluru L: Cervical cancer: Biomarkers for diagnosis and treatment. Clin Chim Acta 445: 7-11, 2015.

4 Wentzensen $\mathrm{N}$ and von Knebel Doeberitz $\mathrm{M}$ : Biomarkers in cervical cancer screening. Dis Markers 23(4): 315-330, 2007.

5 Syrjanen KJ: Immunohistochemistry in assessment of molecular pathogenesis of cervical carcinogenesis. Eur J Gynaecol Oncol 26(1): 5-19, 2005.

6 Kjaer SK, van den Brule AJ, Paull G, Svare EI, Sherman ME, Thomsen BL, Suntum M, Bock JE, Poll PA and Meijer CJ: Type specific persistence of high risk human papillomavirus (HPV) as indicator of high- grade cervical squamous intraepithelial lesions in young women: population-based prospective follow up study. BMJ 325(7364): 572, 2002.

7 Takeda M, Sakuragi N, Okamoto K, Todo Y, Minobe S, Nomura E, Negishi H, Oikawa M, Yamamoto $R$ and Fujimoto S: Preoperative serum SCC, CA125, and CA19-9 levels and lymph node status in squamous cell carcinoma of the uterine cervix. Acta Obstet Gynecol Scand 81(5): 451-457, 2002.

8 Bolli JA, Doering DL, Bosscher JR, Day TG Jr., Rao CV, Owens K, Kelly B and Goldsmith J: Squamous cell carcinoma antigen: clinical utility in squamous cell carcinoma of the uterine cervix. Gynecol Oncol 55(2): 169-173, 1994.

9 Kainz C, Gitsch G, Kohlberger P, Reinthaller A, Kolbl H and Breitenecker G: [Human papillomavirus infections and carcinoembryonic antigen expression in cervix intra-epithelial neoplasia of intermediate grade]. Pathologe 14(5): 264-266, 1993.

10 Tolino A, Ronsini S, Gallo FP, de Conciliis B, Riccio S and Montemagno U: Carcinoembryonic antigen in genital infections caused by HPV. Rev Fr Gynecol Obstet 85(12): 698-701, 1990 (in French).

11 Borras G, Molina R, Xercavins J, Ballesta A and Iglesias J: Tumor antigens CA 19.9, CA 125, and CEA in carcinoma of the uterine cervix. Gynecol Oncol 57(2): 205-211, 1995.

12 Lein M, Jung K, Laube C, Hubner T, Winkelmann B, Stephan C, Hauptmann S, Rudolph B, Schnorr D and Loening SA: Matrix-metalloproteinases and their inhibitors in plasma and tumor tissue of patients with renal cell carcinoma. Int J Cancer 85(6): 801-804, 2000 . 
$13 \mathrm{Li} \mathrm{Y,} \mathrm{Wu} \mathrm{T,} \mathrm{Zhang} \mathrm{B,} \mathrm{Yao} \mathrm{Y} \mathrm{and} \mathrm{Yin} \mathrm{G:} \mathrm{Matrix} \mathrm{metallo-}$ proteinase-9 is a prognostic marker for patients with cervical cancer. Med Oncol 29(5): 3394-3399, 2012.

14 de Brito OM and Scorrano L: Mitofusin 2: a mitochondriashaping protein with signaling roles beyond fusion. Antioxid Redox Signal 10(3): 621-633, 2008.

15 Chen H, Detmer SA, Ewald AJ, Griffin EE, Fraser SE and Chan DC: Mitofusins Mfn1 and Mfn2 coordinately regulate mitochondrial fusion and are essential for embryonic development. J Cell Biol 160(2): 189-200, 2003.

16 Eura Y, Ishihara N, Oka T and Mihara K: Identification of a novel protein that regulates mitochondrial fusion by modulating mitofusin (Mfn) protein function. J Cell Sci 119(Pt 23): 49134925, 2006.

17 Chen KH, Guo X, Ma D, Guo Y, Li Q, Yang D, Li P, Qiu X, Wen S, Xiao RP and Tang J: Dysregulation of HSG triggers vascular proliferative disorders. Nat Cell Biol 6(9): 872-883, 2004.

18 Bach D, Pich S, Soriano FX, Vega N, Baumgartner B, Oriola J, Daugaard JR, Lloberas J, Camps M, Zierath JR, Rabasa-Lhoret $\mathrm{R}$, Wallberg-Henriksson $\mathrm{H}$, Laville M, Palacin M, Vidal H, Rivera F, Brand $\mathrm{M}$ and Zorzano A: Mitofusin-2 determines mitochondrial network architecture and mitochondrial metabolism. A novel regulatory mechanism altered in obesity. J Biol Chem 278(19): 17190-17197, 2003.

19 Zuchner S, Mersiyanova IV, Muglia M, Bissar-Tadmouri N, Rochelle J, Dadali EL, Zappia M, Nelis E, Patitucci A, Senderek J, Parman Y, Evgrafov O, Jonghe PD, Takahashi Y, Tsuji S, Pericak-Vance MA, Quattrone A, Battaloglu E, Polyakov AV, Timmerman V, Schroder JM and Vance JM: Mutations in the mitochondrial GTPase mitofusin 2 cause Charcot-Marie-Tooth neuropathy type 2A. Nat Genet 36(5): 449-451, 2004.

20 Chien KR and Hoshijima M: Unravelling Ras signals in cardiovascular disease. Nat Cell Biol 6(9): 807-808, 2004.

21 Hernandez-Alvarez MI, Thabit H, Burns N, Shah S, Brema I, Hatunic M, Finucane F, Liesa M, Chiellini C, Naon D, Zorzano A and Nolan JJ: Subjects with early-onset type 2 diabetes show defective activation of the skeletal muscle PGC1 \{alpha\}/Mitofusin-2 regulatory pathway in response to physical activity. Diabetes Care 33(3): 645-651, 2010.

22 Wang W, Xie Q, Zhou X, Yao J, Zhu X, Huang P, Zhang L, Wei $\mathrm{J}$, Xie $\mathrm{H}$, Zhou $\mathrm{L}$ and Zheng $\mathrm{S}$ : Mitofusin-2 triggers mitochondria $\mathrm{Ca}^{2+}$ influx from the endoplasmic reticulum to induce apoptosis in hepatocellular carcinoma cells. Cancer Lett 358(1): 47-58, 2015.
23 Jin B, Fu G, Pan H, Cheng X, Zhou L, Lv J, Chen G and Zheng S: Anti-tumour efficacy of mitofusin-2 in urinary bladder carcinoma. Med Oncol 28(Suppl 1): S373-380, 2011.

24 Zhang GE, Jin HL, Lin XK, Chen C, Liu XS, Zhang Q and Yu JR: Anti-tumor effects of MFN2 in gastric cancer. Int J Mol Sci 14(7): 13005-13021, 2013.

25 Fang CL, Sun DP, Chen HK, Lin CC, Hung ST, Uen YH and Lin KY: Overexpression of mitochondrial GTPase MFN2 represents a negative prognostic marker in human gastric cancer and its inhibition exerts anticancer effects. J Cancer 8(7): 1153-1161, 2017.

26 Lou Y, Li R, Liu J, Zhang Y, Zhang X, Jin B, Liu Y, Wang Z, Zhong $\mathrm{H}$, Wen $\mathrm{S}$ and Han B: Mitofusin-2 overexpresses and leads to dysregulation of cell cycle and cell invasion in lung adenocarcinoma. Med Oncol 32(4): 132-143, 2015.

27 Zhang X, Zheng Z, Shin YK, Kim KY, Rha SY, Noh SH, Chung $\mathrm{HC}$ and Jeung HC: Angiogenic factor thymidine phosphorylase associates with angiogenesis and lymphangiogenesis in the intestinal-type gastric cancer. Pathology 46(4): 316-324, 2014.

28 Feng X, Zhu K, Liu J, Chen J, Tang J, Liang Y, Jin R, Liang X and Cai $X$ : The evaluative value of SEMA3C and MFN2 coexpression detected by immunohistochemistry for prognosis in hepatocellular carcinoma patients after hepatectomy. Onco Targets Ther 9: 3213-3221, 2016.

29 Xu K, Chen G, Li X, Wu X, Chang Z, Xu J, Zhu Y, Yin P, Liang $\mathrm{X}$ and Dong L: MFN2 suppresses cancer progression through inhibition of mTORC2/AKT signaling. Sci Rep 7: 41718, 2017.

30 Raaijmakers JH and Bos JL: Specificity in Ras and Rap signaling. J Biol Chem 284(17): 10995-10999, 2009.

$31 \mathrm{Lu} \mathrm{L}$, Wang J, Wu Y, Wan P, Yang G: Rap1A promotes ovarian cancer metastasis via activation of ERK/p38 and notch signaling. Cancer Med 5: 3544-3554, 2016.

32 Girotti MR, Fernandez M, Lopez JA, Camafeita E, Fernandez EA, Albar JP, Benedetti LG, Valacco MP, Brekken RA, Podhajcer OL and Llera AS: SPARC promotes cathepsin Bmediated melanoma invasiveness through a collagen I/alpha2beta1 integrin axis. J Invest Dermatol 131(12): 2438$2447,2011$.

Received March 23, 2018

Revised April 13, 2018

Accepted April 18, 2018 